\title{
Association of Serum Adiponectin with Age, BMI and Other Breast Cancer Biomarkers
}

Rabindra Nath Das ${ }^{1,2 *}$ and Youngjo Lee ${ }^{2}$

${ }^{1}$ Department of Statistics, The University of Burdwan, Burdwan, West Bengal, India

${ }^{2}$ Department of Statistics, College of Natural Science, Seoul National University, Seoul, Korea

\begin{abstract}
Throughout the world, the most commonly observed cancer in women is breast cancer. Low serum adiponectin levels are positively associated with breast cancer, so it is treated as a breast cancer biomarker. The report focuses the association of adiponectin with age, body mass index, and other breast cancer biomarkers. It is derived herein that mean adiponectin is negatively associated with Age $(P=0.0006)$, Body mass index (BMI) $(P<0.0001)$, Resistin $(P=0.0237)$, while it is positively associated with interaction effects Age ${ }^{*} B M I(P=0.0017)$ and $B M I^{*}$ Resistin $(P=0.0615)$. Some partially significant effects such as Leptin (mean model) and Age (variance model) are also included in the model as confounder (known in Epidemiology). Adiponectin is higher for younger women with low body mass index and low resistin levels, and it is not associated with serum glucose and insulin levels.
\end{abstract}

Keywords: Adiponectin; Breast cancer; Leptin; Resistin; Nonconstant variance

\section{Introduction}

Serum adiponectin is a protein hormone which modulates many metabolic processes, along with fatty acid oxidation and glucose regulation $[1,2]$. It is secreted from adipose tissue (and also from placenta in pregnancy) into the bloodstream, and it is very abundant in plasma relative to many hormones [2]. Due to high obesity, serum adiponectin levels are reduced, and it is increased after severe weight loss [3,4]. Low levels of adiponectin are associated with insulin resistance, hyperinsulinaemia, and breast cancer risk [5-7]. Obesity is positively associated with the mortality, incidence and prevalence of breast cancer [3]. Obese breast cancer patients have a higher risk of larger tumors, lymph node metastasis, and higher mortality rates than non-obese patients [4]. The relationship between BMI and risk of breast cancer has been well established [8-10]. Some research articles have shown that adiponectin and leptin have significant correlation with insulin and HOMA-IR [11-13]. Most of the earlier results are based on simple correlation, simple, multiple, Logistic regression analysis which are not appropriate for examining the association of any two variables for a multivariate non-normal heteroscedastic positive continuous data set.

The earlier findings related to the association of adiponectin with age, BMI, glucose, insulin, and other breast cancer biomarkers invite many doubts and debates. The association of adiponectin can only be identified through appropriate modeling of it with age, BMI, glucose, insulin, and other breast cancer biomarkers, which has been little studied in medical literature, considering the original nature of the data set. The present report tries to focus the real associations of adiponectin with age, BMI, glucose, insulin and many breast cancer biomarkers through probabilistic modeling.

\section{Material and Statistical Methods}

\section{Materials}

The report examines the association of adiponectin with age, BMI, glucose, insulin, and other breast cancer biomarkers based on 116 (64 patients and 52 healthy controls) subjects including 10 (1 attribute and 9 continuous) study variables, and the data set is available in the UCI Machine Learning Repository. The study population, data collection process and covariates have been clearly expressed [14]. These are not redisplayed herein. Some research articles are related with the Wisconsin Breast Cancer Dataset (WBCD), Wisconsin Prognosis Breast Cancer (WPBC), and Wisconsin Diagnosis Breast Cancer (WDBC) data sets [15-17]. For ready presenting the covariates in the article, they are reproduced as Age (AGE) (years), Glucose (GCS) $(\mathrm{mg} / \mathrm{dL})$, BMI $\left(\mathrm{kg} / \mathrm{m}^{2}\right)$, Insulin (ISL) $(\mu \mathrm{U} / \mathrm{mL})$, Leptin (LPT) $(\mathrm{ng} /$ mL), HOMA, Adiponectin (ANT) ( $\mu \mathrm{g} / \mathrm{mL}), \mathrm{MCP}-1$ (MCP) (pg/dL), Resistin (RST) (ng/mL), Types of subjects (TOS) (1=Healthy controls; $2=$ Patients)

\section{Statistical methods}

The report aims to derive the relationship of adiponectin with age, BMI, glucose, insulin, and other breast cancer biomarkers using appropriate probabilistic modeling. The aimed response adiponectin is positive heteroscedastic continuous and non-normally distributed random variable. It can be modeled by adopting suitable transformation when the variance is stabilized with the transformation. If the transformation fails, it can be modeled by joint generalized linear models (JGLMs) with Gamma and Log-normal distributions [18-22]. These have not been reproduced in the report. For elaborate ideas about JGLMs, readers may go through $[18,21]$. The response adiponectin has been modeled using both the Log-normal and Gamma JGLMs, and they give identical results. Therefore, these two models are shortly reproduced herein.

Log-normal JGLMs: Let us a consider a positive continuous dependent random variable $y_{i}$ 's with $E\left(y_{i}\right)=\mu_{i}$ (mean parameters), and non-constant variance $\sigma_{i}^{2}$ (dispersion parameters), with $\operatorname{Var}\left(\mathrm{Y}_{\mathrm{i}}\right)=$ $\sigma_{i}^{2} \mu_{\mathrm{i}}^{2}=\sigma_{i}^{2} V\left(\mu_{i}\right)$ say. Generally, the $\log$ transformation $\mathrm{Z}_{\mathrm{i}}=\log \left(\mathrm{Y}_{\mathrm{i}}\right)$ is

*Corresponding author: Rabindra Nath Das, Department of Statistics, The University of Burdwan, Burdwan, West Bengal, India, Tel: +91-9232638970; E-mail: rabin.bwn@gmail.com

Received December 04, 2018; Accepted December 14, 2018; Published December 22, 2018

Citation: Das RN, Lee Y (2018) Association of Serum Adiponectin with Age, BMl and Other Breast Cancer Biomarkers. J Blood Lymph 8: 233. doi: 10.4172/2165 7831.1000233

Copyright: (c) 2018 Das RN, et al. This is an open-access article distributed unde the terms of the Creative Commons Attribution License, which permits unrestricted use, distribution, and reproduction in any medium, provided the original author and source are credited. 
used to stabilize the variance $\operatorname{Var}\left(\mathrm{Z}_{\mathrm{i}}\right) \approx \sigma_{i}^{2}$, but the variance may not be stabilized always. For determining improved model, JGLMs for the mean and variance are adopted. For Log-normal distributed positive random variable $Y_{i}$, with $Z_{i}=\log Y_{i}$, JGLM of the mean and variance are given by

$$
\begin{aligned}
& E\left(Z_{i}\right)=\mu_{z i} \text { and } \operatorname{Var}\left(Z_{i}\right)=\sigma_{z i}{ }^{2}, \\
& \mu_{z i}=x_{i}^{t} \beta \text { and } \log \left(\sigma_{z i}{ }^{2}\right)=g_{i}^{t} \gamma,
\end{aligned}
$$

Where $\mathrm{x}_{i}^{\mathrm{t}}$ and $\mathrm{g}_{\mathrm{i}}^{\mathrm{t}}$ are the vectors of explanatory variables associated respectively, along with the regression coefficients $\beta$ (mean model parameters) and $\gamma$ (variance model parameters).

Gamma JGLMs: For a continuous positive random response $\mathrm{y}_{\mathrm{i}, \mathrm{if}}$ if $E\left(y_{i}\right)=\mu_{i}$ and $\operatorname{Var}\left(y_{i}\right)=\sigma_{i}^{2} V\left(\mu_{i}\right)$, where $\mu_{\mathrm{i}}$ 's and $\sigma_{i}^{2}$ 's are respectively, mean and dispersion parameters, and $V(\cdot)$ reveals the variance function with two parts (in GLM) such as $\sigma_{i}^{2}$ (free of mean changes) and $V\left(\mu_{i}\right)$ (depends on the mean changes). It is noted that the GLM family distribution is located by $V\left(\mu_{i}\right)$, as it is Poisson if $V(\mu)=\mu$, Gamma if $V(\mu)=\mu^{2}$, and Normal if $V(\mu)=1$, etc. So, the Gamma JGLMs of mean and dispersion (when $V(\mu)=\mu^{2}$ ) are

$$
\eta_{i}=g\left(\mu_{i}\right)=x_{i}^{t} \beta \text { and } \varepsilon_{i}=h\left(\sigma_{i}^{2}\right)=w_{i}^{t} \gamma,
$$

where $g(\cdot)$ and $h(\cdot)$ are GLM link functions related to the mean and variance linear predictors respectively, and $x_{i}^{t}, w_{i}^{t}$ are the vectors of explanatory factors/variables, connected to the mean and dispersion parameters respectively. Practically, the maximum likelihood (ML) and the restricted ML (REML) method are used respectively, for estimating the mean and dispersion parameters [17].

\section{Statistical and Graphical Analysis}

The response adiponectin is identified as heteroscedastic which is not stabilized by any suitable transformation. It is considered as the response variable, and the remaining other 9 factors are adopted as the explanatory variables. Adiponectin has been modeled by JGLMs considering both Log-normal and Gamma distributions. The best fitted models have been selected based on the smallest value of Akaike information criterion (AIC) (within each class) which minimizes both the squared error loss and predicted additive errors [23]. Three partially significant factors such as Leptin and interaction effect BMI ${ }^{\star}$ Leptin (in mean model) and age (in variance model) are adopted in the final models as confounder. It is known that partially significant factors adopted in the model are called confounder in Epidemiology. Both the Log-normal and Gamma model analyses outcomes are presented in Table 1. Log-normal (AIC=673.8) and Gamma $(\mathrm{AIC}=673.368)$ fits show similar results (Table 1) based on AIC rule.

All possible valid interpretations are obtained from the data generated probabilistic model considering it as the approximately correct model of the true unknown model. So, the developed model is to be examined using model diagnostic tools. From Table 1, AIC of Gamma fitted models is little smaller than Log-normal, so model checking is adopted to the Gamma fitted models (Table 1), which is displayed in Figure 1. The absolute residuals are plotted against the Gamma fitted values (Table 1) in Figure 1a, which is approximately linear, indicating that variance is constant with the running means. Figure $1 \mathrm{~b}$ displays the normal probability plot for the Gamma fitted mean model (Table 1), which presents no lack of fit. Hence, both the figures support that the fitted Gamma models (Table 1) are approximately same to the unknown true models.

\section{Results}

The summarized form JGLMs fitted outcomes for both Log-normal and Gamma models are displayed in Table 1. The following outcomes are related to the Gamma fitted models in Table 1. It is derived herein that mean adiponectin is negatively associated with Age $(\mathrm{P}=0.0006)$, Body mass index (BMI) $(\mathrm{P}<0.0001)$, Resistin $(\mathrm{P}=0.0237)$ and interaction effect $\mathrm{BMI}^{\star}$ Leptin $(\mathrm{P}=0.1729)$ (partially significant), while it is positively associated with Leptin $(\mathrm{P}=0.1202)$ (partially significant), interaction effects Age ${ }^{\star} \mathrm{BMI}(\mathrm{P}=0.0017)$ and $\mathrm{BMI}{ }^{\star}$ Resistin $(\mathrm{P}=0.0615)$. Variance of adiponectin is partially positively significantly associate with Age $(\mathrm{P}=0.1233)$.

Gamma fitted Adiponectin mean $(\hat{\mu})$ model (from Table 1 ) is $\hat{\mu}=\exp$ (6.7778-0.0617 Age-0.1475 BMI+0.0020 Age ${ }^{\star} \mathrm{BMI}+0.0282$ Leptin-0.0662 Resistin $+0.0018 \mathrm{BMI}^{\star}$ Resistin $-0.0008 \mathrm{BMI}^{\star}$ Leptin), and Gamma fitted Adiponectin variance $\left(\hat{\sigma}^{2}\right)$ model is $\hat{\sigma}^{2}=\exp$ $(-2.318+0.017$ Age $)$.

These two equations present the mean and variance relationship of Adiponectin. It is noted that mean Adiponectin is expressed by Age, BMI, Leptin, Resistin, Age ${ }^{\star}$ BMI, BMI ${ }^{\star}$ Resistin, BMI ${ }^{\star}$ Leptin, while variance of Adiponectin is explained by only Age. Note that adiponectin is modeled by JGLMs. Here mean \& variance both are modeled simultaneously by iterative method [18]. The significance

\begin{tabular}{|c|c|c|c|c|c|c|c|c|c|}
\hline \multirow[t]{2}{*}{ Model } & \multirow[t]{2}{*}{ Covariate } & \multicolumn{4}{|c|}{ Log-normal fit } & \multicolumn{4}{|c|}{ Gamma fit } \\
\hline & & Estimate & S.E. & t-value & P-value & Estimate & S.E. & t-value & P-value \\
\hline \multirow[t]{8}{*}{ Mean } & Constant & 6.8667 & 1.0012 & 6.858 & $<0.0001$ & 6.7778 & 0.9853 & 6.879 & $<0.0001$ \\
\hline & AGE & -0.0627 & 0.0178 & -3.525 & 0.0006 & -0.0617 & 0.0174 & -3.550 & 0.0006 \\
\hline & BMI & -0.1512 & 0.0367 & -4.122 & $<0.0001$ & -0.1475 & 0.0361 & -4.087 & $<0.0001$ \\
\hline & AGE*BMI & 0.0020 & 0.0006 & 3.067 & 0.0027 & 0.0020 & 0.0006 & 3.214 & 0.0017 \\
\hline & LPT & 0.0217 & 0.0184 & 1.179 & 0.2409 & 0.0282 & 0.0180 & 1.566 & 0.1202 \\
\hline & RST & -0.0701 & 0.0296 & -2.371 & $0 . .0195$ & -0.0662 & 0.0289 & -2.293 & 0.0237 \\
\hline & BMI*RST & 0.0020 & 0.0010 & 2.003 & 0.0476 & 0.0018 & 0.0010 & 1.886 & 0.0615 \\
\hline & BMI*LPT & -0.0006 & 0.0006 & -0.976 & 0.3312 & -0.0008 & 0.0006 & -1.372 & 0.1729 \\
\hline \multirow[t]{2}{*}{ Dispersion } & Constant & -2.325 & 0.6674 & -3.483 & 0.0007 & -2.318 & 0.6595 & -3.515 & 0.0006 \\
\hline & AGE & 0.019 & 0.0114 & 1.690 & 0.0939 & 0.017 & 0.0112 & 1.553 & 0.1233 \\
\hline \multicolumn{2}{|c|}{ Above are the final models } & \multicolumn{4}{|c|}{$A I C=673.8$} & \multicolumn{4}{|c|}{$A I C=673.368$} \\
\hline \multicolumn{10}{|c|}{ Additional two variables Glucose \& Insulin are included below to examine their significance } \\
\hline \multirow[t]{2}{*}{ Mean } & Glucose & -0.0011 & 0.0029 & -0.379 & 0.7054 & -0.0015 & 0.0029 & -0.536 & 0.5930 \\
\hline & Insulin & 0.0052 & 0.0057 & 0.914 & 0.3627 & 0.0034 & 0.0056 & 0.609 & 0.5438 \\
\hline
\end{tabular}
of regression coefficients are tested by t-statistic, as usual multiple regression analysis.

Table 1: Results for mean and dispersion models for Adiponectin from Log-Normal and Gamma fit. 

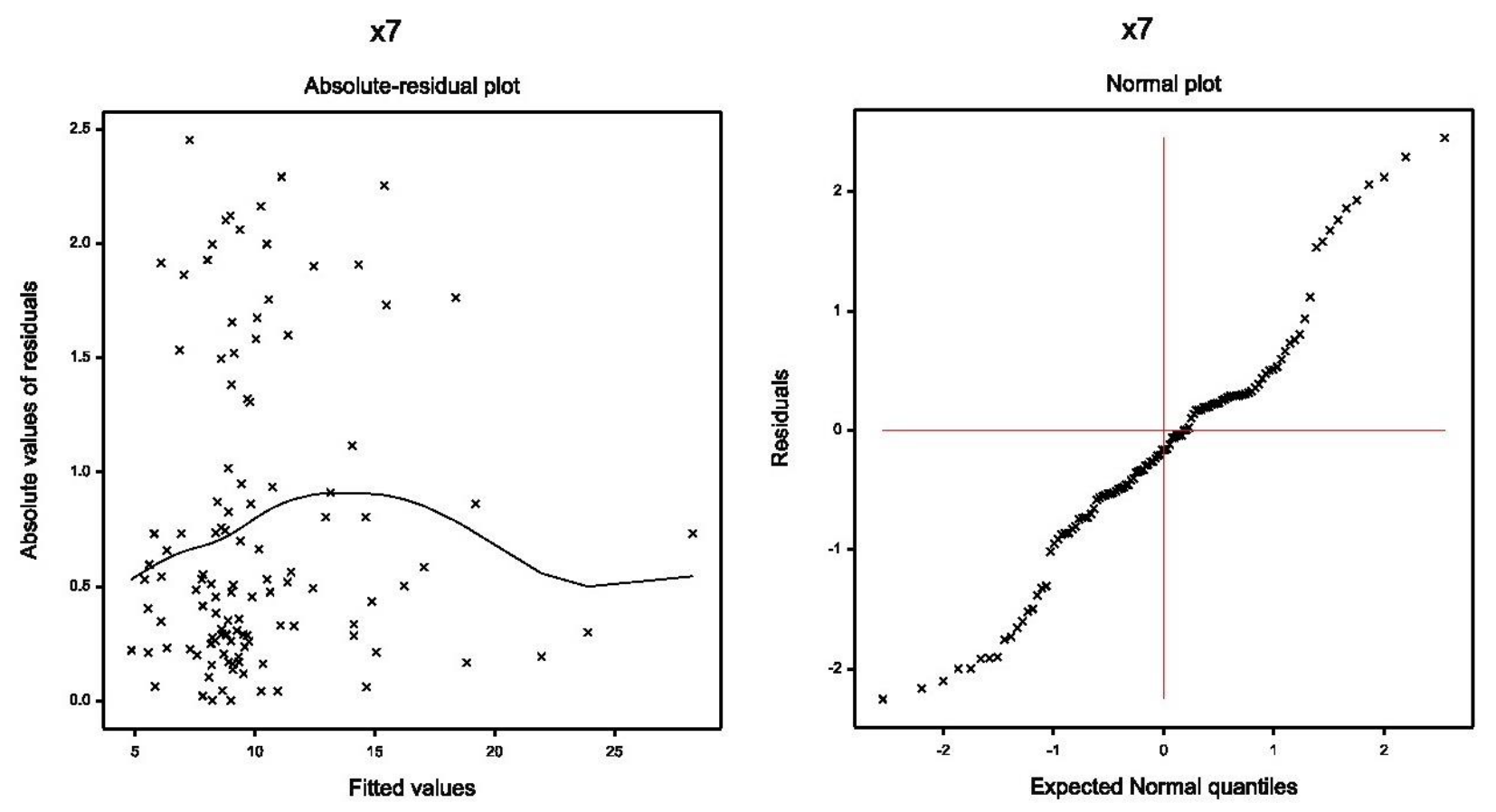

Figure 1: For the joint Gamma fitted models of Adiponectin (Table 1), the (a) absolute residuals plot with respect to the fitted values, and (b) the normal probability plot for the mean model.

\section{Discussion}

Final fitted results for both the distributions are given in Table 1, and also the Gamma fitted mean and variance models of Adiponectin are given above. These two models of Adiponectin (Table 1) conclude the following.

- The mean Adiponectin (ANT) (MANT) is negatively associated with AGE $(\mathrm{P}=0.0006)$, indicating that $\mathrm{ANT}$ is higher for younger women than older. This is observed in practice.

- MANT is negatively associated with BMI $(\mathrm{P}<0.0001)$, concluding that ANT is higher for women with lower BMI. This is also observed in practice.

- MANT is positively associated with the interaction effect $\mathrm{AGE}^{\star} \mathrm{BMI}(\mathrm{P}=0.0017)$, indicating that $\mathrm{ANT}$ increases as the interaction effect $\mathrm{AGE}^{\star} \mathrm{BMI}$ increases. Note that both the marginal effects AGE \& BMI are negatively associated, while their interaction effect is positively associated with ANT.

- MANT is partially positively associated with Leptin (LPT) $(\mathrm{P}=0.1202)$, interpreting that ANT increases as LPT increases.

- MANT is negatively associated with Resistin (RST) $(\mathrm{P}=0.0237)$, implying that ANT increases as RST decreases.

- MANT is positively associated with the interaction effect $\mathrm{BMI}^{*} \mathrm{RST}(\mathrm{P}=0.0615)$, indicating that ANT increases as the interaction effect $\mathrm{BMI}^{\star} \mathrm{RST}$ increases. Note that both the marginal effects BMI \& RST are negatively associated, while their interaction effect is positively associated with ANT.

- MANT is partially negatively associated with the interaction effect $\mathrm{BMI}^{\star} \mathrm{LPT}(\mathrm{P}=0.1729)$, indicating that ANT increases as the interaction effect BMI ${ }^{\star} \mathrm{LPT}$ decreases. Note that the marginal effect BMI is negatively, while LPT is positively, but their interaction effect is negatively associated with ANT.

- Variance of Adiponectin is partially positively associated with Age $(\mathrm{P}=0.1233)$, concluding that Adiponectin variance is higher for older women than younger.

Interpretations of the Adiponectin analysis outcomes have been displayed above. The associations of age, BMI, breast cancer biomarkers such as Leptin and Resistin are shown with Adiponectin. It is found herein that Adiponectin levels are higher for younger women with lower BMI, lower Resistin levels and higher Leptin levels. It is derived herein that lower age, lower BMI, and their join interaction effect (AGE*BMI) increase Adiponectin. Similarly, it is also derived herein that lower BMI, lower Resistin, and their join interaction effect $\left(B M{ }^{\star}{ }^{R S T}\right)$ increase Adiponectin. Note that Adiponectin has no association with glucose levels, insulin levels, MCP-1 and HOMA. Many articles have pointed that Adiponectin has association with glucose and insulin levels, but the present findings have shown that Adiponectin has no association with them (last two rows are added in Table 1 to show their association) $[2,11,12,14]$. The report has derived many joint interaction effects, which have not been pointed in earlier reports. In addition, there are a few similar earlier studies, so it is very difficult to compare the present findings with the earlier findings. For the given data set [14], adiponectin level is insignificant for the subject type. The report is unable to focus any association of adiponectin with many diabetes markers and cardiac factors, as the considered data set [14] does not contain such characters.

\section{Conclusion}

The associations of Adiponectin with age, BMI and other breast cancer biomarkers have been derived herein with probabilistic modeling. Mean and variance models of Adiponectin have been explained by Age, BMI, Leptin, Resistin and their interaction effects. The final mean and variance models of Adiponectin have selected based on comparison of 
the Log-normal and Gamma distributions, lowest AIC value, model checking plots, and small standard error of the estimates (Table 1). Therefore, the final fitted models are approximately correct to the true unknown models, and the interpretations which have been drawn based on the fitted models are exactly true. In addition, the findings support the real situations and some earlier results. Hence, research should have greater faith on these results than those emanating from simple correlation, simple, multiple and Logistic regression models. The report has derived that Adiponectin levels are higher for younger women, with lower BMI, lower Resistin levels \& higher Leptin levels. It is derived herein that lower age, lower BMI, and their join interaction effect (AGE`BMI) increase Adiponectin. Similarly, it is also derived herein that lower BMI, lower Resistin, and their join interaction effect $\left(B M{ }^{*} R S T\right)$ increase Adiponectin. In addition, the report has shown that Adiponectin has no association with glucose, insulin, MCP-1 and HOMA.

The associations (Table 1) of Adiponectin with age, BMI and other breast cancer biomarkers are valid for the data set in [14]. For different similar data sets, fitted models may be different (with respect to regression coefficients), but the conclusions may be identical. It is not examined herein as we have not similar data sets. Moreover, the data set [14] does not contain diabetes markers 2-hours post plasma glucose, HbAlc and random plasma glucose. The reported data set does not contain many diabetes markers and cardiac factors. Future research reports may examine the associations of Adiponectin with all possible diabetes, cardiac and breast cancer biomarkers along with age and BMI. These studies will present the interrelationship of blood biochemical parameters with diabetes, breast cancer and cardiac diseases.

The report has shown that Adiponectin has no association with glucose and insulin (last two additional rows in Table 1). These are contradictions to earlier findings $[2,11,12,14]$. The report has supported some earlier findings (association between Adiponectin and BMI). Many interaction effects have been identified which have not been reported in any previous articles. Medical practitioners may be able to know the different influential factors of Adiponectin from the report. Every woman should reduce her BMI, and care should be taken on Adiponectin levels at older ages.

\section{Conflict of Interest}

The authors confirm that this article content has no conflict of interest.

\section{Acknowledgement}

The authors are very much indebted to the referees who have provided valuable comments to improve this paper. This research was supported by the Brain Research Program through the National Research Foundation of Korea (NRF) funded by the Ministry of Science, ICT and Future Planning (2014M3C7A1062896)

\section{References}

1. Kelesidis I, Kelesidis T, Mantzoros CS (2006) Adiponectin and cancer: A systematic review. Br J Cancer 94: 1221-1225.

2. Schmidt S, Monk JM, Robinson LE, Mourtzakis M (2015) The integrative role of leptin, oestrogen and the insulin family in obesity-associated breast cancer: Potential effects of exercise. Obes Rev 16: 473-487.

3. Lorincz AM, Sukumar S (2006) Molecular links between obesity and breas cancer. EndocrRelat Cancer 13: 279-292.
4. Rose DP, Vona-Davis L (2010) Interaction between menopausal status and obesity in affecting breast cancer risk. Maturitas 66: 33-38.

5. Miyoshi Y, Funahashi T, Kihara S, Taguchi T, Tamaki Y, et al. (2003) Association of serum adiponectin levels with breast cancer risk. Clin Cancer Res 9: 5699-5704.

6. Chen DC, Chung YF, Yeh YT, Chaung HC, Kuo FC, et al. (2006) Serum adiponectin and leptin levels in Taiwanese breast cancer patients. Cancer Lett 237: 109-114

7. Verkasalo PK, Thomas HV, Appleby PN, Davey GK, Key TJ (2001) Circulating levels of sex hormones and their relation to risk factors for breast cancer: a cross-sectional study in 1092 pre- and postmenopausal women (United Kingdom). Cancer Causes Control 12: 47-59.

8. Morimoto LM, White E, Chen Z, Chlebowski RT, Hays J, et al. (2002) Obesity, body size, and risk of postmenopausal breast cancer: the Women's Health Initiative (United States). Cancer Causes Control 13: 741-751.

9. Hirose K, Tajima K, Hamajima N, Takezaki T, Inoue M, et al. (2001) Association of family history and other risk factors with breast cancer risk among Japanese premenopausal and postmenopausal women. Cancer Causes Control 12 349-358.

10. Tung HT, Tsukuma H, Tanaka H, Kinoshita N, Koyama Y, et al. (1999) Risk factors for breast cancer in Japan, with special attention to anthropometric measurements and reproductive history. Jpn J Clin Oncol 29: 137-146.

11. Awadhi SAA, Khaldi RMA, Rammah TA, Kapila K, Mojiminiyi OA (2012) Associations of adipokines \& insulin resistance with sex steroids in patients with breast cancer. Indian J Med 135: 500-505.

12. Danthala M, Rajesh GR, Gundeti S, Raju GS, Chandran P, et al. (2018) Obesity and breast cancer: Association of serum adiponectin, leptin, and adiponectinleptin ratio as risk biomarkers. Indian J Med Paediatr Oncol 39: 292-296.

13. Miyoshi Y, Funahashi T, Kihara S, Taguchi T, Tamaki Y, et al. (2003) Association of Serum Adiponectin Levels with Breast Cancer Risk. Clin Cancer Res 9: 5699-5704.

14. Patrício M, Pereira J, Crisóstomo J, Matafome P, Gomes M, et al. (2018) Using Resistin, glucose, age and BMI to predict the presence of breast cancer. BMC Cancer 18: 18-29.

15. Wolberg WH, Mangasarian OL (1990) Multi surface method of pattern separation for medical diagnosis applied to breast cytology. Proc Natl AcadSci USA 87: 9193-9196.

16. Zheng BC, Yoon SW, Lam SS (2014) Breast cancer diagnosis based on feature extraction using a hybrid of K-means and support vector machine algorithms. Expert Syst Appl 41: 1476-1482.

17. Maglogiannis I, Zafiropoulos E, Anagnostopoulos I (2009) An intelligen system forautomated breast cancer diagnosis and prognosis using SVM basedclassifiers. Appl Intell 30: 24-36.

18. Lee Y, Nelder JA, Pawitan Y (2006) Generalized Linear Models with Random Effects (Unified Analysis via H-likelihood). London: Chapman \& Hall 2006.

19. Das RN, Lee $Y$ (2009) Log-normal versus gamma models for analyzing data from quality-improvement experiments. Quality Engineering 21: 79-87.

20. Das RN, Lee $Y(2010)$ Analysis strategies for multiple responses in quality improvement experiments. International Journal of Quality Engineering and Technology 1: 395-409.

21. Das RN (2014) Robust Response Surfaces, Regression, and Positive Data Analyses. London: Chapman \& Hall 2014.

22. Das RN, Lee Y, Bhattacharyya G (2018) Effects of Plasma Volume and Red Cell Index on Cardiac Parameters for Shock Patients. J Blood Lymph 8: 228.

23. Hastie T, Tibshirani R, Friedman J (2001) The Elements of Statistical Learning Springer-Verlag. 